\title{
Inherited metabolic disorders of glycoconjugate metabolism
}

\author{
Pakanova $Z^{1}$, Nemcovic $M^{1}$, Ziburova $\mathrm{J}^{1,2}$, Mucha $\mathrm{J}^{1}$, Salingova $\mathrm{A}^{3}$, Sebova $\mathrm{C}^{3}$, \\ Jurickova $\mathrm{K}^{4}$, Barath $\mathrm{P}^{1}$ \\ Center of Excellence for Glycomics, Institute of Chemistry, Slovak Academy of Sciences, Bratislava, Slovakia. \\ zuzana.pakanova@savba.sk
}

\begin{abstract}
Inherited metabolic disorders of glycoconjugate metabolism include congenital disorders of glycosylation (CDG) - disorders in biosynthesis of glycoconjugates; and some of the lysosomal storage diseases (LSD) - disorders of their degradation. This review summarizes the brief characteristics of metabolic pathways of synthesis and catabolism of glycoconjugates as well as the latest update of relevant enzymatic defects discovered in population. Every year the number of known subtypes of these disorders dramatically increases as a result of high-throughput analytical infrastructure applied. However, due to the broad spectrum of unspecific clinical symptoms, many patients remain undiagnosed or have wrong diagnosis with ineffective treatment. Thus, disorders of glycoconjugate metabolism should be considered and ruled out in any unexplained syndrome. The collaboration between scientists and physicians plays an important role in the progress of such personalized diagnostics, that is essential mainly for rare diseases (Tab. 2, Fig. 1, Ref. 49). Text in PDF www.elis.sk KEY WORDS: congenital disorders of glycosylation, lysosomal storage disorders.
\end{abstract}

\section{Introduction}

During the last decades, genome and proteome were considered as the major instrument of knowledge of living organisms. Nowadays, science forwarded from genomics and proteomics to glycomics and not only the genetic code and its expression are essential factors of life. Post-translational modifications of proteins, including glycosylation, play a vital role in eukaryotes. Constantly increasing knowledge about pathological changes in glycoprofiles and its integration into genomic and proteomic data opens up new possibilities in the diagnosis, prevention or monitoring of diseases, and also the development of new therapeutic approaches.

\section{Biosynthesis of glycans}

Oligosaccharides are bound to protein via serine or threonine (O-linked oligosaccharides) or asparagine (N-linked oligosac-

${ }^{1}$ Center of Excellence for Glycomics, Institute of Chemistry, Slovak Academy of Sciences, Bratislava, Slovakia, ${ }^{2}$ Department of Clinical Genetics, St. Elizabeth Cancer Institute, Bratislava, Slovakia, ${ }^{3}$ Center for Inherited Metabolic Disorders, National Institute of Children's Diseases, Bratislava, Slovakia, and ${ }^{4}$ Center for Inherited Metabolic Disorders, Department of Pediatrics, National Institute of Children's Diseases and Comenius University Medical School, Bratislava, Slovakia

Address for correspondence: $\mathrm{Z}$. Pakanova, $\mathrm{PhD}$, Center of Excellence for Glycomics, Institute of Chemistry, Slovak Academy of Sciences, Dubravska cesta 9, SK-845 38 Bratislava, Slovakia.

Phone: +421.2.59410213, Fax: +421.2.59410222

Acknowledgement: This work was supported by Ministry of Health of the Slovak Republic under the project registration number 2019/7-CHÚSAV-4, VEGA 2/0130/18 and „Technical infrastructure for biomedical research“, ITMS 26230120008, supported by the Research \& Development Operational Programme funded by the ERDF. charides) (Montreuil et al, 1995). O-glycosylation is a sequential process of transmission of single monosaccharide units and Nglycosylation is more complex, including many partial synthetic and trimming reactions. If a mutation in any enzyme involved in glycan biosynthesis occurs, it may lead to the pathway disruption, demonstrated also with altered phenotype. The complete loss of $\mathrm{N}$-glycosylation is lethal (Freeze and Westphal, 2001).

During the last decades, the relationship between aberrant glycosylation and a variety of pathological conditions, including chronic inflammatory diseases (Verhelst et al, 2020), immunodeficiencies (Ravell et al, 2020), diabetes (Rudman et al, 2019), cardiovascular disorders (Gudelj and Lauc, 2018) or cancer (Gao et al, 2020), was observed. Abnormalities in the glycosylation patterns have been discovered also in patients with Alzheimer's disease (Regan et al, 2019). Nowadays primary disorders in the biosynthesis of O-linked oligosaccharides, N-linked oligosaccharides, and glycans present in glyco-phosphatidylinositols and glycosphingolipids are known (Topaz et al, 2004, Freeze and Aebi, 2005; Almeida et al, 2006).

\section{Catabolism of glycoconjugates}

Catabolic reactions of glycoproteins and glycoconjugates take place in lysosomes as a part of normal metabolic homeostasis and turnover. Their degradation takes place in two opposite directions. The first is a sequential removal of monosaccharides by specific exoglycosidases and the second direction is proteolytic cleavage of the bond between carbohydrate and protein (Winchester, 2005). The final products of glycoconjugate catabolism (monosaccharides, amino acids and dipeptides) are transported across the membrane into the cytosol. Disorders in degradation pathways occurring in lysosomes are classified as lysosomal storage disorders. 


\section{Congenital disorders of glycosylation}

In 1997 only three defects in genes involved in the glycosylation process were known. Nowadays, due to the continuous development of modern analytical methods, more than 130 unique subtypes of these monogenic diseases are known (Post and Lefeber, 2019). The incidence and prevalence of all types of CDG in aggregate have not been well established yet as it is believed that many cases go unrecognized or misdiagnosed, making it difficult to determine their true frequency. The estimated prevalence in European and African American populations is 1: 10,000 based on carrier frequencies of known pathogenic variants in 53 genes (Chang et al, 2018, Jaeken and Matthijs, 2001, Jaeken and Matthijs, 2007). As the serum glycoproteins contain mostly N-linked complex type oligosaccharides, most of the identified CDG defects were observed in the N-glycosylation pathway (Marquardt and Denecke, 2003). In approximately $20 \%$ of CDG patients the specific enzyme defect is still not described, and these undiagnosed patients are classified as the CDG subtype $\mathrm{x}$. Summary of known CDG subtypes with the list of deficient enzymes/proteins and corresponding OMIM numbers is shown in the Table 1.

\section{Clinical manifestations and diagnostics of CDG}

Congenital disorders of glycosylation are a rapidly expanding group of genetic diseases (Grubenmann et al, 2003). This group is characterized by various clinical manifestations with multisystem phenotype that includes disorders in central and peripheral nervous system, often associated with disorders of coagulation, and endocrinologic findings (Peng et al, 2020, Jaeken and Matthijs, 2001). Approximately $20 \%$ of patients do not survive beyond five years of age due to widespread organ dysfunction (Jones et al, 2013). Common clinical manifestations associated with the CDG include delayed development and decreased intellect, ataxia, seizures, retinopathy, growth disorders, cardiomyopathy, pericardial effusions, endocrine abnormalities, renal dysfunction, hepatic failure, disorders of bone development and decreased mineralization, late closure of fontanel, cutis laxa (Morava et al, 2008), skeletal dysplasia (Coman et al, 2007), high frequency of early mortality and typical dysmorphia. Facial dysmorphia is demonstrated by a high forehead, triangular face, large ears, strabismus, and a thin upper lip. Peripheral dysmorphia is manifested by abnormal fat distribution and inverted nipples (Jaeken and Matthijs, 2007).

Wide symptomatology of CDG and structural diversity of glycoconjugates ranks among the diseases as difficult to diagnose (Grubenmann et al, 2004). Currently, as a selective screening test, isoelectric focusing (IEF) of serum transferrin (Tf) is established in many countries (De Jong and Van Eijk, 1988). Its abnormal carbohydrate-deficient isoforms are characteristic biochemical marker for CDG (Stibler, Jaeken. 1990), but this method does not provide sufficient resolution needed to distinguish the specific enzymatic defect. Representative IEF Tf profiles of CDG, compared to the healthy serum, are shown in Figure 1.

To improve the diagnostic possibilities, several methods utilizing mass spectrometry, due to its sensitivity and specificity, were

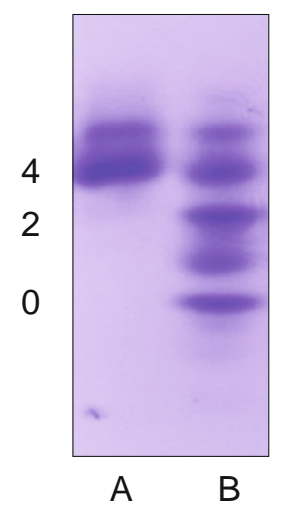

Fig. 1. IEF profiles of serum transferrin isoforms which are divided by the number of sialic acids. A- Negative sample, B- PMM2 CDG sample, 0,2,4- numbers of terminal sialic acids attached. The PMM2CDG patients present asialo-Tf and in large quantities disialo-Tf; on the other hand, tetrasialo-Tf in PMM2-CDG serum is observed in a lower abundance compared to the healthy serum.

developed. They are widely used to elucidate the structure of the glycans (Barbosa et al, 2019) or glycoproteins. In recent years, broader accessibility and higher rates of conclusive diagnoses have made next-generation sequencing (NGS) one of the preferred approaches for the solving of undiagnosed genetic disorders and the first gene panel targeted for CDGs was released in 2010 (Jones et al, 2013). The advantage of NGS lies in its robust approach, that is usually required in cases where there is no information about what single gene in such complex glycosylation pathway could be defective.

As the advanced methods mentioned above are not suitable for routine diagnostics, comprehensive collaboration between the clinical geneticists, physicians and researchers is essential. In eligible process, after the disorder of glycoconjugate metabolism is suspected, samples of serum or urine are sent to the centers of inherited metabolic disorders where the selective screening is performed. If the result from screening is positive, borderline or ambiguous, samples can be further analyzed by personalized approaches to characterize the specific biomarkers, determine their levels; or to precisely locate the mutations and predict their further impact.

\section{Lysosomal storage disorders}

Lysosomal storage disorders (LSDs) are a group of over 50 genetic metabolic diseases, leading to deficiency of a lysosomal enzyme, activator, transport protein; or, in some cases, the nonlysosomal protein included in lysosomal biogenesis. The result of deficient activity of lysosomal catabolic enzymes is the accumulation of metabolites in these organelles (Futerman and Van Meer, 2004). Disruption of one pathway can lead to many clinical symptoms, depending on the location of the disorder. LSDs are classified by the type of metabolite that accumulates: lipidoses, mucopolysaccharidoses, glycoproteinoses, sphingolipidoses, multi-enzymatic deficiencies, disorders of lysosomal transport and 
Tab. 1. Summary of the CDG subtypes (edited from Denecke. 2009; Uemura et al, 2008; Chang et al, 2018; Peanne et al, 2018; Sparks et al, 2017).

\begin{tabular}{|c|c|c|}
\hline CDG subtype & Deficient enzyme/protein & OMIM* \\
\hline ALG1-CDG & GDP-Man:GlcNAc2-PP-dolichol mannosyltransferase & 608540 \\
\hline ALG2-CDG & GDP-Man:Man1GlcNAc2-PP-dolichol mannosyltransferase & 607906 \\
\hline ALG3-CDG & Dolichyl-P-Man: Man5GlcNAc2-PP-dolichol mannosyltransferase & 601110 \\
\hline ALG6-CDG & Dolichyl-P-Glc:Man9GlcNAc2-PP-dolichol glucosyltransferase & 603147 \\
\hline ALG8-CDG & Dolichyl-P-Glc:Glc1Man9GlcNAc2-PP-dolichol- $\alpha$-1,3-glucosyltransferase & 608104 \\
\hline ALG9-CDG & Dolichyl-P-Man: $\alpha$-1,2-mannosyltransferase & 608776 \\
\hline ALG11-CDG & Asparagine-linked glycosylation protein 11 & 613661 \\
\hline ALG12-CDG & Dolichyl-P-Man:Man7GlcNAc2-PP-dolichol mannosyltransferase & 607143 \\
\hline ALG13-CDG & ALG13 UDP-N-Acetylglucosaminyltransferase subunit & 300884 \\
\hline ALG14-CDG & ALG14 UDP-N-Acetylglucosaminyltransferase subunit & 616227 \\
\hline ATP6V0A2-CDG & ATPase, $\mathrm{H}+$ transporting, lysosomal, $\mathrm{V} 0$ subunit A2 & 219200 \\
\hline B3GLCT-CDG & $\beta$-3-Glucosyltransferase & 261540 \\
\hline B4GALNT1-CDG & $\beta-1,4-\mathrm{N}-$ Acetylgalactosaminyltransferase 1 & 609195 \\
\hline B4GALT1-CDG & Golgi UDP-Gal:GlcNAc $\beta$-1,4-galactosyltransferase & 607091 \\
\hline$\overline{\mathrm{CAD}-\mathrm{CDG}}$ & Carbamoyl phosphate synthetase/Aspartate transcarbamoylase/Dihydroorotase (CAD trifunctional protein) & 616457 \\
\hline CCDC115-CDG & Coiled-coil domain-containing protein 115 & 616828 \\
\hline COG1-CDG & Golgi conserved oligomeric complex & 611209 \\
\hline COG2-CDG & Golgi conserved oligomeric complex & 617395 \\
\hline COG4-CDG & Golgi conserved oligomeric complex & 613489 \\
\hline COG5-CDG & Golgi conserved oligomeric complex & 613612 \\
\hline COG6-CDG & Golgi conserved oligomeric complex & 606977 \\
\hline COG7-CDG & Golgi conserved oligomeric complex & 608779 \\
\hline COG8-CDG & Golgi conserved oligomeric complex & 611182 \\
\hline DDOST-CDG & Dolichyl-diphosphoooligosacharide-protein glycosyltransferase & 614507 \\
\hline DHDDS-CDG & Dehydrodolichyl diphosphate synthase & 613861 \\
\hline DOLK-CDG & Dolichyl-kinase & 610768 \\
\hline DPAGT1-CDG & UDP-GlcNAc:dolicholphosphate N-acetylglucosamine-1-phosphotransferase & 608093 \\
\hline DPM1-CDG & Dolichyl-P-mannosyltransferase 1, catalytic subunit & 608799 \\
\hline DPM2-CDG & Dolichyl-phosphate mannosyltransferase 2 , regulatory subunit & 615042 \\
\hline DPM3-CDG & Dolichyl-phosphate mannosyltransferase - polypeptide 3 & 612937 \\
\hline EXT1-CDG & Exostosin glycosyltransferase I & 133700 \\
\hline EXT2-CDG & Exostosin glycosyltransferase II & 133701 \\
\hline FCSK-CDG & L-Fucose kinase & 618324 \\
\hline FUCT1-CDG & Golgi GDP-fucose transporter & 266265 \\
\hline FUT8-CDG & $\alpha$-1,6-fucosyltransferase & 618005 \\
\hline GALNT3-CDG & UDP-N-Acetyl- $\alpha$-D-Galactosamine:Polypeptide N-Acetylgalactosaminyltransferase 3 & 211900 \\
\hline GCS1-CDG & Mannosyl oligosaccharide glycosidase (Glucosidase I) & 606056 \\
\hline MAGT1-CDG & Magnesium transporter 1 & 301031 \\
\hline MGAT2-CDG & $\alpha$-1,6-mannosyl-glycoprotein beta-1,2-GlcNAc-transferase & 212066 \\
\hline MPDU1-CDG & Mannose-P-dolichol utilization defect 1 & 609180 \\
\hline MPI-CDG & Mannosophosphate isomerase & 602579 \\
\hline NUS1-CDG & NUS1 dehydrodolichyl diphosphate synthase subunit & 617082 \\
\hline PGM1-CDG; also known as GSD XIV & Phosphoglucomutase 1 & 614921 \\
\hline PIGA-CDG & Phosphatidylinositol glycan anchor biosynthesis class A protein & 300868 \\
\hline PIGL-CDG & Phosphatidylinositol glycan anchor biosynthesis class L protein & 280000 \\
\hline PIGM-CDG & Phosphatidylinositol glycan anchor biosynthesis class M protein & 610293 \\
\hline PMM2-CDG & Phosphomannomutase II & 212065 \\
\hline POFUT1-CDG & Protein-O-fucosyltransferase 1 & 615327 \\
\hline POGLUT1-CDG & Protein-O-glucosyltransferase 1 & 617232 \\
\hline RFT1-CDG & Flipase & 612015 \\
\hline SEC23B-CDG & SEC23 Homolog B, Coat Complex II Component & 616858 \\
\hline SLC35A1-CDG & CMP-Sialic acid transporter & 603585 \\
\hline SLC35A2-CDG & UDP-Galactose transporter & 300896 \\
\hline SLC35C1-CDG & GDP-Fucose transporter & 266265 \\
\hline
\end{tabular}




\begin{tabular}{lll}
\hline CDG subtype & Deficient enzyme/protein & OMIM* \\
\hline SLC39A8-CDG & Solute carrier family 39, member 8 & 616721 \\
\hline SRD5A3-CDG & Steroid 5- $\alpha$-reductase III & 612379 \\
\hline SSR4-CDG & Translocon-associated protein, delta subunit & 300934 \\
\hline ST3GAL3-CDG & $\beta$-Galactoside $\alpha$-2,3-sialyltransferase 3 & 615006 \\
\hline ST3GAL5-CDG & $\beta$-Galactoside $\alpha$-2,3-sialyltransferase 5 & 609056 \\
\hline STT3A-CDG & Oligosaccharidyltransferase complex, catalytic subunit STT3A & 615596 \\
\hline STT3B-CDG & Oligosaccharidyltransferase complex, catalytic subunit STT3B & 615597 \\
\hline TMEM165-CDG & Transmembrane protein 165 & 614727 \\
\hline TMEM199-CDG & Transmembrane protein 199 & 616829 \\
\hline TUSC3-CDG & Tumor suppressor candidate 3 & 611093 \\
\hline CDG I/IIx & Still unknown CDG defect & 212067 \\
\hline * OMIM - Online Mendelian Inheritance in Man - Phenotype MIM Number (www.ncbi.nlm.nih.gov/omim)
\end{tabular}

Tab. 2. Summary of the LSDs subtypes (edited from Winchester, 2005, Vellodi, 2005).

\begin{tabular}{|c|c|c|}
\hline LSD type & Deficient enzyme/protein & OMIM* \\
\hline$\alpha$-Mannosidose & $\alpha$-D-mannosidase & 248500 \\
\hline$\beta$-Mannosidose & $\beta$-D-mannosidase & 248510 \\
\hline Aspartylglucosaminury & Aspartylglucosaminidase & 208400 \\
\hline Cystinosis & Lysosomal cystin transporter & 219800,219900 \\
\hline Fabry disease & $\alpha$-D-galactosidase $\mathrm{A}$ & 301500 \\
\hline Farber disease & Ceramidase & 230800 \\
\hline Fucosidose & $\alpha$-L-fucosidase & 230000 \\
\hline Gaucher disease & Glucosylceramid $\beta$-galactosidase & 230800,230900 \\
\hline GM1-gangliosidosis & $\beta$-D-galactosidase & 230500,230600 \\
\hline Krabb disease & Galactozylceramide $\beta$-galactosidase & 245200 \\
\hline Metachromatic leucodystrophy & Arylsulphatase A & 250100 \\
\hline MPS I - Hurler - Scheie syndrome & $\alpha$-1-Iduronidase & 607015 \\
\hline MPS I - Hurler syndrome & $\alpha$-1-Iduronidase & 607014 \\
\hline MPS I - Scheie syndrome & $\alpha$-1-Iduronidase & 607016 \\
\hline MPS II & Iduronatesulphate sulphatase & 309900 \\
\hline MPS IIIA & Heparan-S-sulphate sulphamidase & 252900 \\
\hline MPS IIIB & N-acetyl-D-glucosaminidase & 252920 \\
\hline MPS IIIC & Acetyl-CoA-glucosaminid N-acetyltransferase & 252930 \\
\hline MPS IIID & $\mathrm{N}$-acetyl-glucosaminine-6-sulphate sulphatase & 252940 \\
\hline MPS IVA & Galactosamine-6-sulphate sulphatase & 253000 \\
\hline MPS IVB & $\beta$-galactosidase & 253010 \\
\hline MPS IX & Hyaluronidase & 601492 \\
\hline MPS VI & Arylsulphatase B & 253200 \\
\hline MPS VII & $\beta$-glucuronidase & 253220 \\
\hline Mucolipidose I (sialidose) & Sialidase & 256550 \\
\hline Mucolipidosis II/IIIa,b & N-acetylglucosaminyl-1-phosphotransferase & 252500,252600 \\
\hline Mucolipidosis IV & Catepsin A & 256540,252650 \\
\hline Neuronal ceroid lipofuscinosis & Palmitoyl protein-thioesterase / tripeptidyl peptidase 1 & 256730 \\
\hline Niemann-Pick disease A, B & Sphingomyelinase & 257200,607616 \\
\hline Niemann-Pick disease C,D & NPC1, NPC2 protein & 257220,607625 \\
\hline Pompe disease & $\alpha$-glucosidase & 232300 \\
\hline Pyknodysostosis & Catepsin K & 265800 \\
\hline Sandhoff disease & $\beta$-hexosaminidase B & 268800 \\
\hline Schindler disease & $\alpha$-D-galactosidase B & 609241 \\
\hline Tay-Sachs disease & $\beta$-hexosaminidase $\mathrm{A}$ & 272800 \\
\hline Wolman disease & Lysosomal acid lipase & 278000 \\
\hline
\end{tabular}

* OMIM - Online Mendelian Inheritance in Man (www.ncbi.nlm.nih.gov/omim) 
760-766

other diseases related to disorders of lysosomal proteins (Vellodi, 2005, Winchester, 2005).

According to prevalence, LSDs are, as well as CDGs, classified as rare diseases. The numbers of patients are different depending on the individual type, such as 1: 57,000 newborns in Gaucher disease, or about 1: 4.2 million in sialidosis (Meikle et al, 1999). Gaucher disease is the most prevalent inherited disorder among Ashkenazi Jews with carrier frequency of about $6 \%$ (Bronstein et al, 2014). The overall prevalence of LSDs in some regions, according to the available literature, is reaching up to $1: 7,000-8,000$ newborns (Meikle et al, 1999; Poorthuis et al, 1999). Overview of LSDs subtypes with the list of defect enzymes/proteins and OMIM numbers is summarized in Table 2.

It is important to mention that not all of the LSDs are involved in the catabolism of glycoconjugates and not all disorders of glycoconjugate catabolism are of lysosomal origin. The first congenital disorder of deglycosylation (CDDG) was described in 2013 (Freeze, 2013), caused by the disruption in NGLY1 gene (coding peptide $\mathrm{N}$-glycanase), is an enzyme deficiency predicted to cause accumulation of $\mathrm{N}$-glycosylated proteins in the cytoplasm and possible endoplasmic reticulum stress. Accumulation of the undegraded material in the cytoplasm may have additional toxic effects (Enns et al, 2014), comparable to the accumulation of substrates in lysosomes, typical for LSDs. Although this disorder is nowadays considered as CDG, it has typically normal Tf IEF profile and on the other hand, abnormal oligosaccharides present in the urine of patients, that is common for LSDs.

\section{Clinical manifestations and diagnostics of LSDs}

Each LSD subtype is characteristic by different clinical and pathological profile that is associated with the accumulated substrate. Some defects may have its different phenotypic variance explained by the residual enzyme activities, but usually patients with the same mutation exhibit widely differing clinical signs; and may even be asymptomatic. Common clinical signs of LSD are abnormalities of bone development, organomegaly and disorders of central nervous system (Mehta et al, 2006). Disorders in glycoconjugate metabolism are characterized by a progressive onset that often led to the early death of the patient.

Based on a wide variety of clinical symptoms, LSDs, as well as CDGs, were characterized as difficult to diagnose. Diagnostics of LSD is usually focused on the identification of key metabolites that may occur in abnormal levels in various body fluids. The first step in diagnostic workup generally consists of urinary analyses of specific undegraded molecules. One of these is thin layer chromatography (TLC) of oligosaccharides present in patient's urine. Another diagnostic approach is based on the determination of enzyme activities in dried blood spots, that can be performed as the selective screening for suspected cases; or as general newborn screening (Gelb et al, 2019). However, enzymatic activity assays are not available for all of LSD types and their significant disadvantage is the fact that carriers of these mutations cannot be determined, because the enzyme activities of carriers correlate with normal subjects (Wang et al, 2011).
As mentioned above, a common feature of all the LSDs is specific metabolite accumulation. Therefore, mass spectrometry and NMR spectroscopy-based methods are a potential tool for fast, accurate and non-invasive diagnosis detecting metabolites excreted in the urine of patients. The last proper step to confirm the diagnosis is analysis of DNA by NGS or simple Sanger sequencing, when there is clear indication for single genetic defect. In addition to diagnostics, advanced analytical techniques may provide subsidiary information about the treatment efficacy through precise monitoring of levels of biomarkers (Pakanova et al, 2018).

\section{Treatment of inherited metabolic disorders of glycoconjugate metabolism}

Up to this date, no effective treatments for the majority of CDGs were developed. For some subtypes, dietary supplementation therapies, such as oral administration of mannose for MPICDG (Niehues and Hasilík. 2000) and fucose for SLC35C1-CDG (Marquardt et al, 1999); or pharmacological chaperones for CDGs that are classified as misfolding disorders (Andreotti et al, 2015, Brasil et al, 2018), were reported as successful and improving the condition of patients. The discovery of appropriate therapeutic approaches in the treatment of CDGs still remains a major issue, since the prevalence as well as the number of these unique diseases is increasing. Never before have so many disorders from the same family been identified in such short time lapse (Jaeken. 2010). Since the prevalence of individual LSDs is significantly higher than that of CDGs, the number of available and effective therapies is more favorable. Up to this date, twenty-three orphan drugh were FDA-approved for the treatment of 12 LSDs (updated from Garbade et al, 2020). Treatment of LSDs is focused mainly on substrate- or enzyme- replacement therapies, chaperones and small molecules. These data suggest more positive perspective for the development of treatment of disorders in glycoconjugate catabolism than in the case of disorders in their synthesis.

Since the disorders of glycoconjugate metabolism are a group of monogenic diseases, they are potential candidates for gene therapy. Nevertheless, vector-targeted immune responses remain a major limitation of this gene-delivery tool in clinical practice. The reduced number of subjects suitable for clinical trials might also cause the current low number of curative treatments (Brasil et al, 2018).

\section{Conclusion}

This review provides a brief summary of knowledge of biosynthesis or degradation of glycoconjugates, known defects in their metabolic pathways and their clinical and biochemical effects in human organism. Nowadays, the amount of glycan structures correlating with various phenotypes is rapidly increasing, and the diagnosis and treatment of these diseases still remain a challenge. Thus, the collaboration between research laboratories, physicians and clinical institutions, where the screening for hereditary metabolic diseases is performed, plays an important role in the progress of diagnostics of CDGs and LSDs subtypes as well as in the development in new therapeutic approaches. 


\section{References}

1. Almeida AM, Murakami Y, Layton D M et al. Hypomorphic promoter mutation in PIGM causes inherited glycosylphosphatidylinositol deficiency. Nat Med 2006; 12: 846-851.

2. Andreotti G, Monti MC, Citro V et al. Heterodimerization of Two Pathological Mutants Enhances the Activity of Human Phosphomannomutase2. PLoS One 2015; 10 (10): e0139882.

3. Barbosa EA, Fontes NC, Santos SCL et al. Relative quantification of plasma N-glycans in type II congenital disorder of glycosylation patients by mass spectrometry. Clin Chim Acta 2019; 492: 102-113.

4. Brasil S, Pascoal C, Francisco R et al. CDG Therapies: From Bench to Bedside. Int J Mol Sci 2018; 19 (5): 1304.

5. Bronstein S, Karpati M, Peleg L. An update of Gaucher mutations distribution in the Ashkenazi Jewish population: prevalence and country of origin of the mutation R496H. Isr Med Assoc J 2014; 16 (11): 683-685.

6. Chang IJ, He M, Lam CT. Congenital disorders of glycosylation. Ann Transl Med 2018; 6 (24): 477.

7. Coman D, Bostock D, Hunter M et al. Primary skeletal dysplasia as a major manifesting feature in an infant with congenital disorder of glycosylation type Ia. J Inherit Metab Dis 2007; 30 (Suppl 1): 65.

8. De Jong G, Van Eijk HG. Microheterogenity of human serum transferrin: a biological phenomenon studied by isoelectric focusing in immobilised pH gradients. Electrophoresis. 1988; 9: 589-98.

9. Denecke J. Biomarkers and diagnosis of congenital disorders of glycosylation. Expert Opin Med Diagn 2009; 3 (4): 395-409.

10. Enns, GM, Shashi V, Bainbridge $M$ et al. Mutations in NGLY1 cause an inherited disorder of the endoplasmic reticulum-associated degradation pathway. Genet Med 2014; 16: 751-8. Erratum: Genet Med.2014; 16: 568.

11. Freeze HH, Aebi M. Altered glycan structures: the molecular basis of congenital disorders of glycosylation. Curr Opin Struct Biol 2005; 15 : 490-498.

12. Freeze HH, Westphal V. Balancing N-linked glycosylation to avoid disease. Biochimie 2001; 83 (8): 791-799.

13. Freeze, H. H. Understanding human glycosylation disorders: biochemistry leads the charge. J Biol Chem 2013; 288: 6936-6945.

14. Futerman AH, Van Meer G. The cell biology of lysosomal storage disorders. Nat Rev Mol Cell Biol 2004; 5: 554-565.

15. Gao T, Wen T, Ge Y et al. Disruption of Core 1-mediated O-glycosylation oppositely regulates CD44 expression in human colon cancer cells and tumor-derived exosomes. Biochem Biophys Res Comm 2020; 521 (2): 514-520.

16. Garbade SF, Zielonka M, Mechler K et al. FDA orphan drug designations for lysosomal storage disorders - a cross sectional analysis. medRxiv 2020; preprint first posted online Jan 10th, 2020.

17. Gelb MH, Lukacs Z, Ranieri E et al. Newborn Screening for Lysosomal Storage Disorders: Methodologies for Measurement of Enzymatic Activities in Dried Blood Spots. Int J Neonatal Screen 2019; 5 (1): 1.

18. Grubenmann CE, Frank CG, Hulsmeier AJ et al. Deficiency of the first mannosylation step in the N-glycosylation pathway causes congenital disorders of glycosylation type Ik. Human Mol Gen 2004; 13 (5): $535-542$.

19. Gudelj I, Lauc G. Protein N-Glycosylation in Cardiovascular Diseases and Related Risk Factors. Curr Cardiovasc Risk Rep 2018; 12, Article 16.
20. Jaeken J. Congenital disorders of glycosylation. Ann NY Acad Sci 2010; 1214: 190-198.

21. Jaeken J, Matthijs G. Congenital disorders of glycosylation. A Rev Genomics Hum Genet 2001; 2: 129-151.

22. Jaeken J, Matthijs G. Congenital disorders of glycosylation: a rapidly expanding disease family. A Rev Genomics Hum Genet 2007; 8: 261-278.

23. Jones MA, Rhodenizer D, da Silva C. Molecular diagnostic testing for congenital disorders of glycosylation (CDG): Detection rate for single gene testing and next generation sequencing panel testing. Mol Gen Met 2013; $110(1-2) ; 78-85$.

24. Marquardt T, Brune T, Luhn K et al. Leukocyte adhesion deficiency II syndrome, a generalised defect in fucose metabolism. J Pediatr. 1999; 134: 681-688.

25. Marquardt T, Denecke J. Congenital disorders of glycosylation: review of their molecular bases, clinical presentations and specific therapies. Eur J Pediatr 2003; 162: 359-379.

26. Mehta A, Beck M, Sunder-Plassmann G et al. History of lysosomal storage diseases: an overview. In: Mehta A, Beck M, Sunder-Plassmann G, editors. Fabry Disease: Perspectives from 5 Years of FOS. Oxford: Oxford PharmaGenesis; 2006.

27. Meikle PJ, Hopwood JJ, Clague AE et al. Prevalence of Lysosomal Storage Disorders. JAMA. 1999; 281 (3): 249-254.

28. Meikle PJ, Hopwood JJ, Clague AE et al. Prevalence of lysosomal storage disorders. JAMA. 1999; 281: 249-254.

29. Montreuil J, Vliegenhart JFG, Schachter H, eds. Glycoproteins. New comprehensive biochemistry, Vol. 29A. Amsterdam: Elsevier, 1995: $644 \mathrm{pp}$.

30. Morava E, Legeber DJ, Urban $\mathbf{Z}$ et al. Defining the phenotype in an autosomal cutis laxa syndrome with a combined Congenital Disorder of Glycosylation. Eur J Hum Genet 2008; 16: 28-35.

31. National Center for Biotechnology Information (US). Genes and Disease [Internet]. Bethesda (MD): National Center for Biotechnology Information (US); 1998-. Gaucher disease.

32. Niehues R, Hasilik M. Carbohydrate-deficient glycoprotein syndrome (CDGS) type Ib. A hereditary metabolic disease and its therapy. MMW Fortschr Med 2000; 142: 171-172.

33. Pakanova Z, Matulova M, Uhliarikova I et al. Case study: monitoring of Glc4 tetrasaccharide in the urine of Pompe patients, use of MALDITOF MS, and 1H NMR. Chem Papers 2018; 73: 701-711.

34. Peanne R, de Lonlay $P$, Foulquier $\mathbf{F}$ et al. Congenital disorders of glycosylation (CDG): Quo vadis? E J Med Gen 2018; 61 (11): 643-663.

35. Peng T, Lv C, Tan $\mathbf{H}$ et al. Novel PMM2 missense mutation in a Chinese family with non-syndromic premature ovarian insufficiency. J Assist Rep Gen 2020; 1-8.

36. Poorthuis BJ, Wevers RA, Kleijer WJ et al. The frequency of lysosomal storage diseases in The Netherlands. Hum Genet. 1999; 105: $151-156$.

37. Post MA, Lefeber D. Clinical glycomics in the diagnostic laboratory. Ann Transl Med 2019; 7 (Suppl 6): S220.

38. Ravell JC, Matsuda-Lennikov M, Chauvin SD et al. Defective glycosylation and multisystem abnormalities characterize the primary immunodeficiency XMEN disease. J Clin Invest 2020; 130 (1): $507-522$. 
760-766

39. Regan P, McClean PL, Smyth T, Doherty M. Early Stage Glycosylation Biomarkers in Alzheimer's Disease. Medicines (Basel) 2019; 6 (3): 92 .

40. Rudman N, Gornik O, Lauc G. Altered N-glycosylation profiles as potential biomarkers and drug targets in diabetes. FEBS Letters 2019; 593: 1598-1615.

41. Sparks SE, Krasnewich DM. Congenital Disorders of N-Linked Glycosylation and Multiple Pathway Overview 2005 Aug 15 [Updated 2017 Jan 12]. In: Adam MP, Ardinger HH, Pagon RA et al., eds. GeneReviews [Internet]. Seattle (WA): University of Washington, Seattle; 1993-2020.

42. Stibler H, Borg S. Evidence of a reduced sialic acid content in serum transferrin in male alcoholics. Alcohol Clin Exp Res. 1981; 5: 545-549.

43. Stibler H, Jaeken J. Carbohydrate deficient serum transferrin in a new systemic hereditary syndrome. Arch Dis Child. 1990; 65: 107-111.

44. Topaz, O, Shurman DL, Bergma, R et al. Mutations in GALNT3, encoding a protein involved in O-linked glycosylation, cause familial tumoral calcinosis. Nat Genet 2004; 36: 579-581.
45. Uemura M, Tamura K, Chung $S$ et al. Novel 5 alpha-steroid reductase (SRD5A3, type-3) is overexpressed in hormone-refractory prostate cancer. Cancer Sci 2008; 99 (1): 81-86.

46. Vellodi A. Lysosomal storage disorders. Br J Haematol 2005; 128 : 413-431.

47. Verhelst X, Dias AM, Colombel JF et al. Protein Glycosylation as a Diagnostic and Prognostic Marker of Chronic Inflammatory Gastrointestinal and Liver Diseases. Gastroent 2020; 158 (1): 95-110.

48. Wang RY, Bodamer OA, Watson MS et al. Lysosomal storage diseases: Diagnostic confirmation and management of presymptomatic individuals. Gen Med 2011; 13 (5): 457-484.

49. Winchester B. Lysosomal metabolism of glycoproteins. Glycobiology $2005 ; 15$ (6): 1R-15R 